\title{
Synthesis of a Highly Aromatic and Planar [10]Annulene
}

\author{
Karnjit Parmar; Christa S. Blaquiere; Brianna E. Lukan; Sydnie N. Gengler; Michel Gravel* \\ Department of Chemistry, University of Saskatchewan, Saskatoon, SK, Canada, S7N 5C9
}

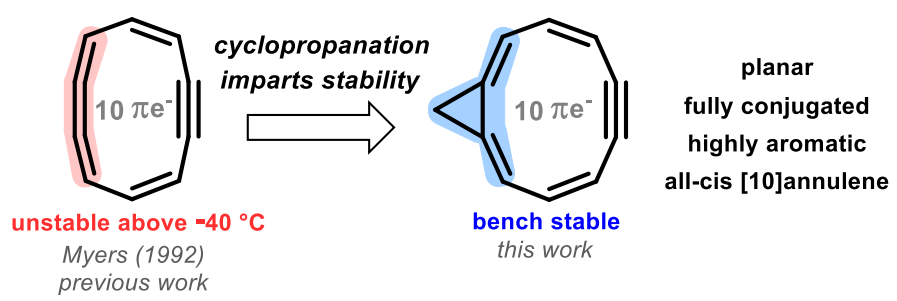

\begin{abstract}
As the next neutral structure following Hückels $(4 n+2) \pi \mathrm{e}^{-}$rule, a planar and aromatic [10]annulene is the ideal framework to study the link between ring size and aromaticity. However, the puckered geometry of the parent [10]annulene (1) suggests that the aromatic stabilization energy (ASE) is not sufficient to overcome the ring strain that exists when the system is forced into planarity. ${ }^{1}$ It has been shown computationally that this ring strain can be alleviated through the addition of two or more cyclopropane rings to the periphery $(5-6),{ }^{2}$ thereby creating theoretically aromatic structures. An alternative strategy to eliminating the issue of ring strain was demonstrated experimentally with the successful preparation of the highly aromatic 1,6-didehydro[10]annulene (2). ${ }^{3}$ However, the system rapidly cyclizes at $-40^{\circ} \mathrm{C}$ to a naphthalene diradical due to the close proximity of the in-plane p-orbitals present in the system. Here we show that cyclopropanating one side of the unstable annulene (2) successfully prevents the destabilizing cross-ring interaction while maintaining a highly aromatic structure. Remarkably, the formed [10]annulene (7) is bench stable and can be stored for extended periods of time.
\end{abstract}

Aromaticity is a fundamental concept that permeates many facets of chemistry. ${ }^{4}$ Identifying (anti)aromatic motifs in various forms has helped explain the (in)stability of both transition state ${ }^{5}$ and ground state structures. ${ }^{6}$ Our working understanding and ability to predict its occurrence has also informed the preparation of materials. ${ }^{7}$ However, despite these advances, we still lack much in the way of a fundamental understanding.

The traditional criteria for aromaticity proposed by Hückel require that a compound be cyclic, planar, conjugated, and contain $(4 n+2) \pi$ electrons. ${ }^{8,9,10}$ Any compound that can satisfy these criteria is predicted by Hückel molecular orbital (HMO) theory to have some degree of aromatic stabilization energy. ${ }^{11}$ Besides the benzenoid archetypes, charged Hückel systems of various ring sizes have also been prepared to explore the effect of ring size on aromaticity. ${ }^{12}$ Unfortunately, the systematic study of these charged species is often quite challenging when compared to neutral annulenes. The larger [18] annulene has been prepared and has been shown to be aromatic, ${ }^{13}$ though its low-frequency out-of-plane vibrations and flexibility in solution make 
it difficult to probe its aromaticity experimentally. ${ }^{14,15}$ There has also been a considerable amount of interest in very large aromatic systems with nanoscale structures, such as those prepared by Anderson et al. ${ }^{16}$

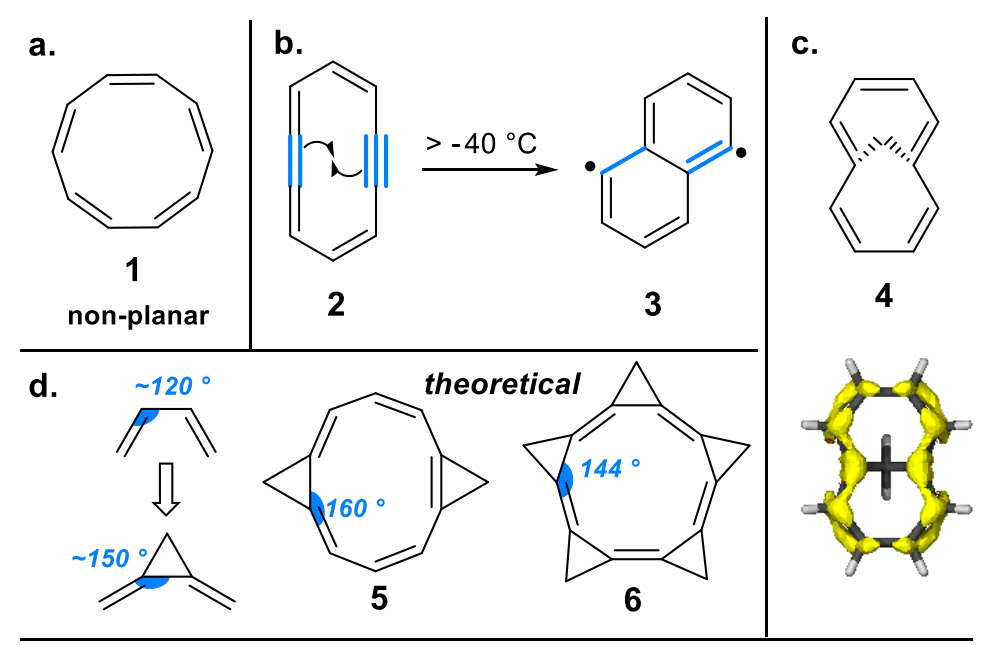

e. our hypothesis:

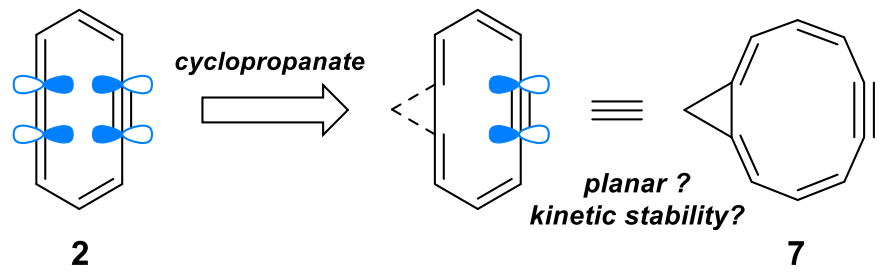

Figure 1: a. The parent [10]annulene (1) has been found to be non-aromatic due to its non-planarity; b. The cyclization of 1,6-didehydro[10]annulene (2) to a biradical (3); c. The structure of 1,6-methano[10]annulene (4) and the unusual anisotropy of the induced current density (AICD) isosurface; $d$. The expansion of CCC bond angles upon cyclopropanation has been shown to generate theoretically planar [10]annulenes (5-6); e. Cyclopropanation of the thermodynamically stable $\mathbf{2}$ may afford a kinetically stable and planar annulene (7) that retains its aromaticity.

As the next highest Hückel aromatic neutral hydrocarbon, a rigid and planar [10]annulene would provide a desirable framework to study the link between aromaticity and ring size. Because the parent [10]annulene (1) is non-aromatic ${ }^{1,17,18}$ (Figure 1a) it must be constrained to force it to adopt a planar conformation that allows for electron delocalization. Myers and coworkers found success in this regard through the preparation of a 1,6-didehydro[10]annulene derivative (2), which has shown evidence of aromaticity. ${ }^{3}$ However, the $\pi$-bonds orthogonal to the conjugated aromatic system readily interact across the ring, resulting in a rapid intramolecular cyclization above $-40{ }^{\circ} \mathrm{C}$ (Figure 1b). The first reported alternant aromatic [10]annulene is the bridged 1,6-methano[10]annulene (4). It was prepared by Vogel in $1964^{19}$ and has recently shown potential as an organic material. ${ }^{7 b}$ The structure is stable and aromatic with a slight deviation from planarity (4, Figure $1 \mathrm{c}$ ). Despite this, it has been shown that the 
electronic structure of $\mathbf{4}$ more closely resembles a homonaphthalene due to cross-ring interactions. ${ }^{20,21}$ Additionally, the presence of trans double bonds as well as the unusual and non-homogenous magnetically induced current also suggests it is not an ideal material to study the aromaticity of expanded benzene-like planar [10]annulenes. ${ }^{22}$

In an effort to identify potential planar [10]annulene frameworks for further study Schleyer et al. explored cyclopropanated derivatives (Figure $1 \mathrm{~d}$ ). ${ }^{2}$ It was shown computationally that cyclopropanated all-cis [10]annulenes (5 and 6) could adopt a planar structure due to the increased exo bond angles of cyclopropenes (Figure $1 \mathrm{~d}$, left). ${ }^{2}$ However, despite their significant predicted aromatic stabilization energy no preparation of a cyclopropanated [10]annulene has been reported to date. Consequently, no experimental information is available regarding their thermodynamic and kinetic stability.

In order to definitively study the role of ring size on aromaticity we sought to identify a bench stable all-cis [10]annulene with a sigma framework more reminiscent of an expanded benzenoid. As the Myers annulene was found to be a thermodynamic minimum, it was believed that cyclopropanation of one of the in-plane $\pi$-bonds of 2 (Figure 1e) would prevent the transannular orbital interaction, thereby creating a kinetically stable structure (7, Figure 1e). This would generate a structure reminiscent of those predicted by Schleyer and one that closely resembles an all-cis and planar [10]annulene.

\section{a. Synthetic strategy towards planar [10]annulene (7)}

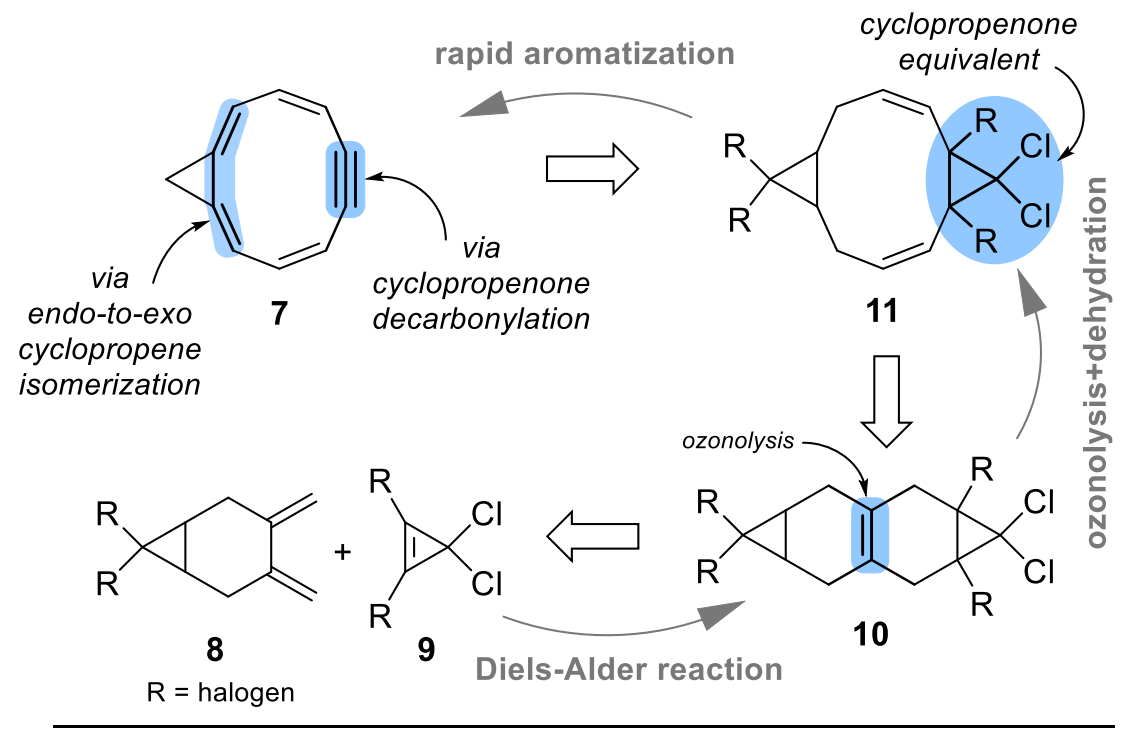

\section{b. Proposed intermediates for a benzocyclopropene synthesis}

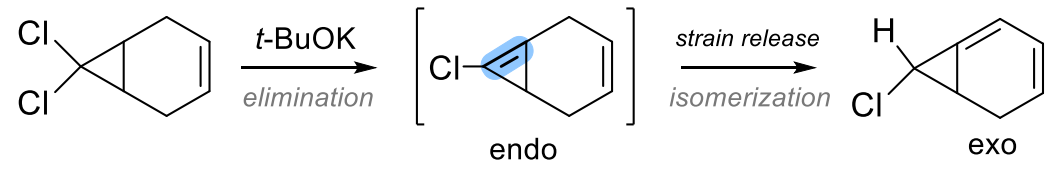

Figure 2: a. A strategy towards the preparation of 7 using a rapid end-stage aromatization procedure; $b$. The proposed mechanism for a benzocyclopropene 
synthesis involves an elimination-isomerization sequence (endo-to-exo cyclopropene). A final elimination-isomerization results in benzocyclopropene (see $\mathrm{SI}$ ).

The essential strategy employed involved constructing a suitably oxidized but strain-free 10-membered ring that could be rapidly aromatized (Figure 2a, 11). A slow and stepwise introduction of strain was rejected in favour of the use of a late-stage rapid sequence of highly reliable strain inducing transformations, bypassing the need to carry unstable and non-aromatic intermediates through the synthesis. As kinetic stability of the final product was a concern, the possibility for a mild aromatization procedure had to be considered. A dichlorocyclopropane group was thought to be beneficial for this purpose as it would improve substrate versatility. Elimination of chloride would generate a transient cyclopropene that would isomerize to an exomethylene cyclopropane, irrespective of an aromatic or conjugative driving force (Figure $2 b$ ). This aromatization step was shown to work for the preparation of cyclopropabenzene (see SI). ${ }^{23}$

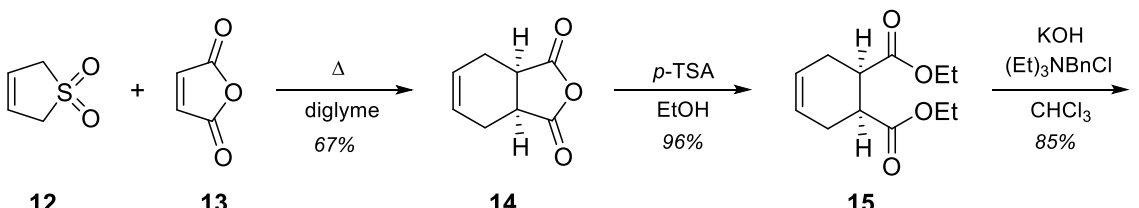

15

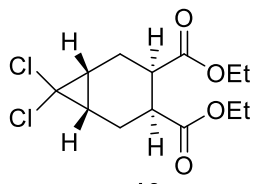

16

17

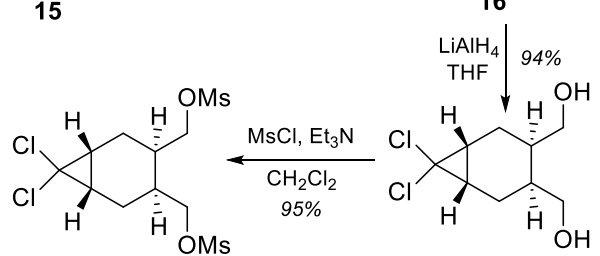

18

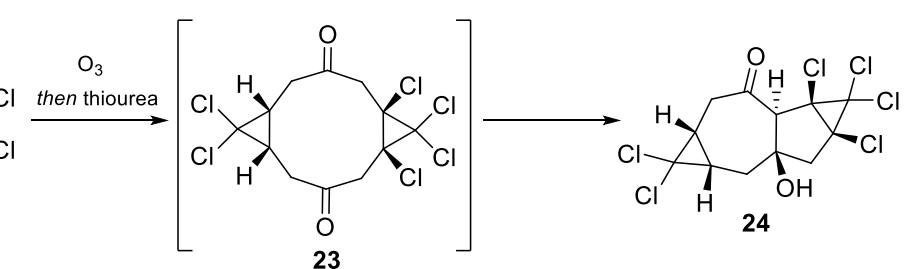

isolable on small scale

X-ray data

Figure 3: Synthetic efforts towards the generation of the suitably functionalized

cyclodecane precursor.

The preparation of diene 19 closely followed literature procedures ${ }^{24}$ with some minor modifications. ${ }^{25}$ The synthesis began with the preparation of 14 via a Diels-Alder reaction between sulfolene (12) and maleic anhydride (13). The adduct (14) could easily be converted to 16 via an ethanolysis and cyclopropanation. $\mathrm{LiAlH}_{4}$ reduction afforded diol 17 , which was readily mesylated to provide 18. Elimination to the diene 19 proceeded with a high yield when it was first converted to the corresponding diiodide (S1, see SI). Exposing the diene 19 to tetrachlorocyclopropene (21) in THF for 3 days generated 22 as a 3:1 ratio of cis and trans 
diastereomers. A subsequent ozonolysis with a standard reductive quench afforded the corresponding diketone $\mathbf{2 3}$, which could be isolated on smaller scales. However, this diketone proved very sensitive and a transannular aldol product (24) was typically obtained when the reaction was scaled up, regardless of the quenching reagent and temperature.
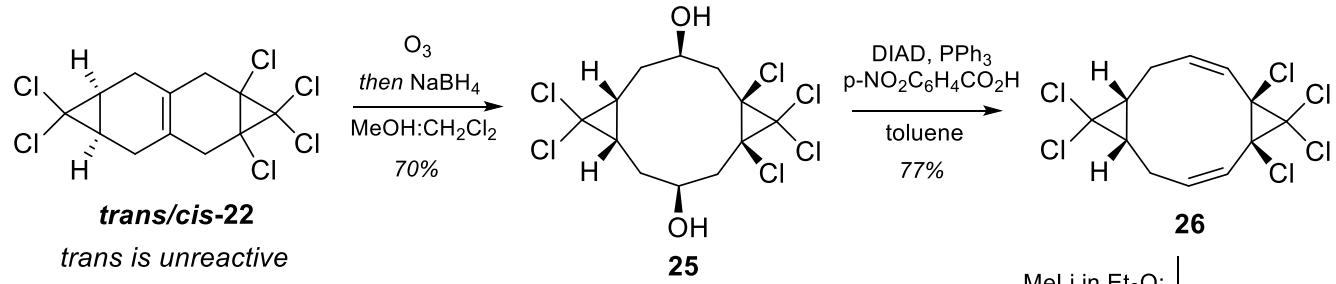

trans is unreactive

25

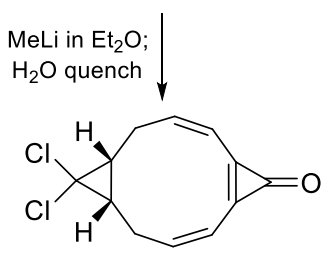

27

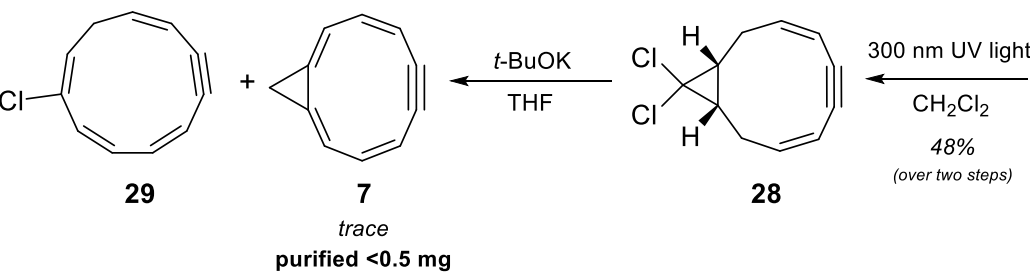

Figure 4: Final preparation of the aromatic [10]annulene 7.

Fortunately, a sodium borohydride quench of the ozonolysis reaction mixture at $-78^{\circ} \mathrm{C}$ was found to generate diol $\mathbf{2 5}$ as a single diastereomer in $\mathbf{7 0 \%}$ yield (Figure 4 ). Note that typically only cis-22 underwent ozonolysis and all attempts to induce an ozonolysis of trans-22 by prolonging the reaction time or increasing the temperature resulted in the formation of an intramolecular aldol product like 24. All attempts to dehydrate diol $\mathbf{2 5}$ by elimination of the corresponding dimesylate only afforded the mono-alkene and decomposition products (not shown). Hoping that the other diastereomer of the diol would yield more favourable results, it was decided to attempt a double Mitsunobu inversion. Unexpectedly and to our delight, the desired diene $\mathbf{2 6}$ was directly obtained in high yield. We speculate that the nucleophile could not approach the concave face of the rigid system, resulting in the more favourable elimination pathway instead. The conditions strongly favoured the symmetric diene 26, presumably due to the strong electron withdrawing nature of the adjacent tetrachlorocyclopropane moiety. ${ }^{26}$ Careful exposure of 26 to MeLi at $0{ }^{\circ} \mathrm{C}$ generated a dichlorocyclopropene (not shown) by a 1,2-didechlorination without noticeable formation of Skatteb $\varnothing \mid$ rearrangement products. ${ }^{27}$ The intermediate dichlorocyclopropene could be hydrolyzed in situ to generate the bench stable cyclopropenone 27.

Decarbonylation of $\mathbf{2 7}$ by exposure to $300 \mathrm{~nm}$ UV light in dilute dichloromethane proceeded smoothly to yield the dienyne $\mathbf{2 8}$. Initial attempts to oxidize $\mathbf{2 8}$ or to carry out a double elimination/isomerization sequence with tBuOK at room temperature led to material degradation. Decreasing the temperature eventually produced observable aromatic signals $(>7$ ppm). These were obtained alongside a major product in the ${ }^{1} \mathrm{H}$ NMR spectrum that was 
identified as $\mathbf{2 9}$. It was presumed that the major product $\mathbf{2 9}$ was obtained by deprotonation of the allylic proton in $\mathbf{2 8}$ with a subsequent cyclopropane ring opening. Interestingly, the crude reaction mixture remained intact over several days and the aromatic signals that were produced persisted despite storage in $\mathrm{CDCl}_{3}$. Unfortunately however, only minute amounts of material could ever be obtained. Purification attempts were further complicated due to the unexpected extreme volatility of $\mathbf{7}$ and the surprisingly similar behaviour of the two products ( $\mathbf{7}$ and $\mathbf{2 9}$ ). The materials were observed to coelute in both normal and reverse phase chromatography and even co-distilled. ${ }^{28}$ Reverse phase HPLC showed peak resolution, but the small amounts of material and its high volatility would have rendered material recovery impractical. Eventually it was discovered that doping a PTLC plate with $\mathrm{AgNO}_{3}$ prior to development resulted in a separation that was sufficient to purify the materials. ${ }^{29}$ As the alkyne in $\mathbf{7}$ is heavily delocalized in the aromatic system, it presumably has a weaker affinity to the $\mathrm{Ag}^{+}$dopant than the alkyne in the non-aromatic 29. Using this technique, the compound could be purified and fully characterized. Interestingly, despite the presence of only trace amounts of material it had a very distinct and pleasant smell. It is also notable that the product is remarkably stable, persisting in $\mathrm{CDCl}_{3}$ for months at $-20^{\circ} \mathrm{C}$ and weeks at room temperature without noticeable degradation.
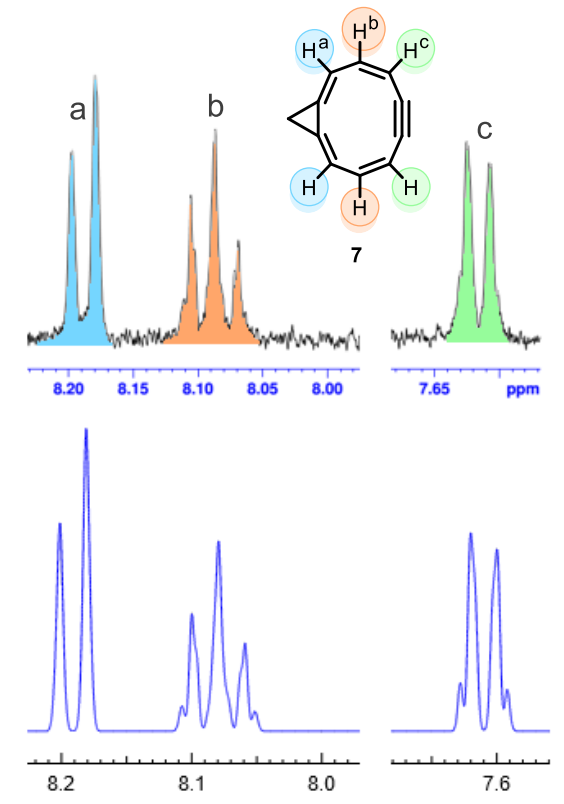

Figure 5: top: experimental spectrum $\left({ }^{1} \mathrm{H}-\mathrm{NMR}\right)$ of the aromatic region of [10]annulene 7; bottom: simulated spectrum $\left({ }^{1} \mathrm{H}-\mathrm{NMR}\right)$ of the aromatic region of [10]annulene 7.

The experimental ${ }^{1} \mathrm{H}$ NMR spectrum of the purified [10]annulene 7 displayed four distinct signals, three of which appeared in the aromatic region between 7.6 and $8.2 \mathrm{ppm}$ (Figure 5, top). ${ }^{30}$ The signals displayed the expected multiplicities with minor broadening. Second order effects and long-range couplings rendered experimental coupling constant extraction 
challenging, although a spectrum obtained with computed chemical shifts and coupling constants very closely matched the signals observed in the aromatic region (Figure 5, bottom). ${ }^{31}$ The close matching computed ${ }^{1} \mathrm{H}-\mathrm{NMR}$ spectrum obtained in the absence of rovibrational considerations suggests it is a highly rigid structure that maintains both planarity and aromaticity. ${ }^{32}$ This is in contrast to other larger aromatic structures such as [18]annulene, whose computed ${ }^{1} \mathrm{H}-\mathrm{NMR}$ spectrum can only be obtained after considering dynamic motion. ${ }^{14 b}$ Nucleus-independent chemical shift (NICS) 33 computations show a highly aromatic system with NICS(1) iso $=-12.0$ and $\operatorname{NICS}(1)_{z z}=-31.9$ (benzene $\mathrm{NICS}_{\text {iso }}=-10.6$ and $\left.\mathrm{NICS}(1)_{z z}=-30.09\right)$. The uniformity of the magnetically induced current isosurface as calculated by the anisotropy of the induced ring current (AICD) method is also quite apparent (Figure 6). ${ }^{34}$ Additionally, all computed $s p^{2}-s p^{2}$ bond lengths fall between $1.37 \AA$ and $1.41 \AA$, further corroborating the high degree of delocalization. Additional minima corresponding to a potential cumulenic structure could not be located, suggesting that the annulene exists as one fully delocalized minimum rather than two interconverting species. Indeed, natural resonance theory ${ }^{35}$ analysis shows dominant contributions from the cumulenic and alkynic valence bond structures with a slight preference for the latter.$^{36}$ Interestingly, the presence of the cyclopropane ring appears to have no effect on the aromatic system, ${ }^{37}$ and induces only a limited amount of strain (see $\mathrm{SI}$ ). ${ }^{38}$ This is consistent with the literature data on benzocyclopropenes where it has been shown repeatedly that such Mills-Nixon effects do not exist. ${ }^{39}$
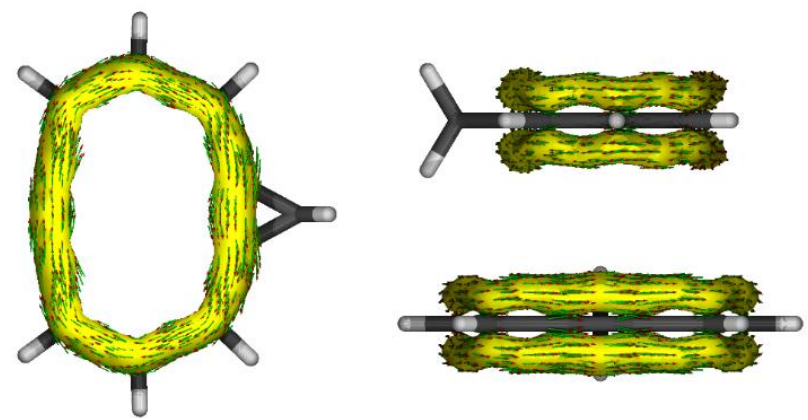

Figure 6: Anisotropy of the induced current density (AICD) map showing a homogenous ring current with an applied magnetic field. The magnetic field is perpendicular to the aromatic ring.

The quest to prepare an aromatic [10]annulene has been a long standing challenge for synthetic chemists. Here we show that it is possible to synthesize the first kinetically and thermodynamically stable all-cis [10]annulene structure by formally cyclopropanating the Myers 1,6-didehydro[10]annulene (2), thus confirming our hypothesis. The planar [10]annulene 7 by all measures is highly aromatic and remains remarkably conjugated despite the presence of both an alkyne and cyclopropane ring. The successful synthesis also supports the indicated mechanism for endo-to-exo cyclopropene isomerization (Figure 2b) ${ }^{40}$ and shows the largely untapped potential of this transformation for generating strained systems. The transformation appears to 
have no dependence on aromaticity and may ultimately prove to be useful in the preparation of other annulenes.

\section{Associated Content}

Experimental procedures and characterization data are provided in the supporting information (SI).

\section{Author Information}

\section{Corresponding Author}

Michel Gravel (University of Saskatchewan)

michel.gravel@usask.ca

\section{Acknowledgment}

We thank the Natural Sciences and Engineering Research Council of Canada (NSERC), the Canada Foundation for Innovation and the University of Saskatchewan for financial support. We would also like to thank the Saskatchewan Structural Sciences Centre (SSSC).

\section{References}

1. (a) Masamune, S.; Darby, N. [10]Annulenes and other (CH)10 hydrocarbons. Accounts of Chemical Research 1972, 5, 272-281; (b) Masamune, S.; Hojo, K.; Hojo, K.; Bigam, G.; Rabenstein, D. L. Geometry of [10]annulenes. Journal of the American Chemical Society 1971, 93, 4966-4968; (c) Masamune, S.; Seidner, R. T. [10]Annulenes. Journal of the Chemical Society D: Chemical Communications 1969, 542-544.

2. Schleyer, P. v. R.; Jiao, H.; Sulzbach, H. M.; Schaefer, H. F. Highly Aromatic Planar all-cis[10]Annulene Derivatives. Journal of the American Chemical Society 1996, 118, 2093-2094.

3. Myers, A. G.; Finney, N. S. Synthesis of 1,6-didehydro[10]annulene. Observation of its exceptionally facile rearrangement to form the biradical 1,5-dehydronaphthalene. Journal of the American Chemical Society 1992, 114, 10986-10987.

4. Schleyer, P. v. R. Introduction: Aromaticity. Chemical Reviews 2001, 101, 1115-1118.

5. Schleyer, P. v. R.; Wu, J. I.; Cossío, F. P.; Fernández, I. Aromaticity in transition structures. Chemical Society Reviews 2014, 43, 4909-4921.

6. See ref 5

7. (a) Perlstein, J. H. "Organic Metals" - The Intermolecular Migration of Aromaticity. Angewandte Chemie International Edition in English 1977, 16, 519-534; (b) Peart, P. A.; Repka, L. M.; Tovar, J. D. Emerging Prospects for Unusual Aromaticity in Organic Electronic Materials: The Case for Methano[10]annulene. European Journal of Organic Chemistry 2008, 2008, 2193-2206.

8. (a) Hückel, E. Zur Quantentheorie der Doppelbindung. Zeitschrift für Physik 1930, 60, 423-456; (b) Hückel, E. Quantentheoretische Beiträge zum Benzolproblem. Zeitschrift für Physik 1931, 70, 204-286; (c) von E. Doering, W.; Detert, F. L. CYCLOHEPTATRIENYLIUM OXIDE. Journal of the American Chemical Society $1951,73,876-877$. 
9. Currently there are many forms of aromaticity that are accepted to exist. Various rules have been predicted and discovered, for example varying rules exist for: excited state aromaticity (Chem. Rev. 2014, 114, 10, 5379-5425), Möbius aromaticity (Chem. Rev. 2005, 105, 3697-3715) spherical aromaticity (Chem. Rev. 2001, 101, 5, 1153-1184), and 3D aromaticity (Org. Lett. 2007, 9, 17, 3263-3266). Other interesting forms of aromaticity include metallaaromaticity (10.1021/acs.chemrev.0c00392) and homoaromaticity (Chem. Rev. 2001, 101, 5, 1185-1204).

10. Spitler, E. L.; Johnson, C. A.; Haley, M. M. Renaissance of Annulene Chemistry. Chemical Reviews 2006, 106, 5344-5386.

11. Aromatic stabilization energy in HMO theory (energy of delocalization) considers the sum total of all orbital energies (each multiplied by their occupancy) subtracted by the sum of orbital energies of a corresponding system with localized double bonds.

Current methods to quantify aromaticity extend past pure energetic criteria. The most commonly accepted quantification measures are typically obtained computationally by considering magnetic criteria (Chem. Soc. Rev., 2015,44, 6597-6615), and electron delocalization measures (Chem. Soc. Rev., 2015, 44, 6434-6451). Structural and energetic criteria are still occasionally used as well (Chem. Rev. 2005, 105, 10, 3773-3811).

12. (a) Breslow, R.; Hover, H.; Chang, H. W. The Synthesis and Stability of Some Cyclopropenyl Cations with Alkyl Substituents. Journal of the American Chemical Society 1962, 84, 3168-3174; (b) Olah, G. A.; Bollinger, J. M.; White, A. M. Stable carbonium ions. LXXXIX. Tetramethylcyclobutenium dication, an aromatic 2 .pi.-electron system. Journal of the American Chemical Society 1969, 91, 3667-3669; (c) Boche, G.; Etzrodt, H.; Marsch, M.; Thiel, W. The Dianion 1,2,3,4-Tetraphenylcyclobutadienediide. Angewandte Chemie International Edition in English 1982, 21, 133-133; (d) Boche, G.; Etzrodt, H.; Marsch, M.; Thiel, W. The Dianion 1,2-Diphenylbenzocyclobutadienediide. Angewandte Chemie International Edition in English 1982, 21, 132-133; (e) Peters, D. 349. The cyclopentadienyl anion. Part I. 1,2Dimethoxycarbonylcyclopentadienylsodium, a stabilised cyclopentadienyl anion. Journal of the Chemical Society (Resumed) 1959, 1757-1760; (f) Von E.Doering, W.; Knox, L. H. The Cycloheptatrienylium (Tropylium) Ion. Journal of the American Chemical Society 1954, 76, 3203-3206; (g) Olah, G. A.; Staral, J. S.; Paquette, L. A. Novel aromatic systems. III. The 1,3,5,7-tetramethylcyclooctatetraene dication, the first 8C-6.pi. Hueckeloid system. Journal of the American Chemical Society 1976, 98, 1267-1269; (h) Katz, T. J. THE CYCLOÖCTATETRAENYL DIANION. Journal of the American Chemical Society 1960, 82, 3784-3785.

13. (a) Sondheimer, F.; Wolovsky, R.; Amiel, Y. Unsaturated Macrocyclic Compounds. XXIII.1 The Synthesis of the Fully Conjugated Macrocyclic Polyenes Cycloöctadecanonaene ([18]Annulene),2 Cyclotetracosadodecaene ([24]Annulene), and Cyclotriacontapentadecaene ([30]Annulene). Journal of the American Chemical Society 1962, 84, 274-284; (b) Ermer, O. Concerning the Structure of [18]Annulene. Helvetica Chimica Acta 2005, 88, 2262-2266; (c) Lungerich, D.; Nizovtsev, A. V.; Heinemann, F. W.; Hampel, F.; Meyer, K.; Majetich, G.; Schleyer, P. v. R.; Jux, N. [18]Annulene put into a new perspective. Chemical Communications 2016, 52, 4710-4713.

14. (a) Baldridge, K. K.; Siegel, J. S. Correlation of Empirical $\delta(T M S)$ and Absolute NMR Chemical Shifts Predicted by ab Initio Computations. The Journal of Physical Chemistry A 1999, 103, 4038-4042; (b) Kwan, E. E.; Liu, R. Y. Enhancing NMR Prediction for Organic Compounds Using Molecular Dynamics. Journal of Chemical Theory and Computation 2015, 11, 5083-5089.

15. On average, [18]annulene is aromatic, but it's relatively large vibrations and conformational flexibility make assessing the reactivity and properties of the most aromatic structure difficult to do. This is clearly seen by the inability to predict the $1 \mathrm{H}-\mathrm{NMR}$ spectrum of [18]annulene without rovibrational considerations.

16. Peeks, M. D.; Claridge, T. D. W.; Anderson, H. L. Aromatic and antiaromatic ring currents in a molecular nanoring. Nature 2017, 541, 200-203. 
17. (a) Van Tamelen, E. E.; Pappas, B. C. T. cis-9,10-Dihydronaphthalene-cyclodecapentaene valence bond isomer system. Journal of the American Chemical Society 1971, 93, 6111-6120; (b) van Tamelen, E. E.; Burkoth, T. L. Cyclodecapentaene. Journal of the American Chemical Society 1967, 89, 151-152; (c) Van Tamelen, E. E.; Burkoth, T. L.; Greeley, R. H. trans-9,10-Dihydronaphthalene-cyclodecapentaene valence bond isomer system. Journal of the American Chemical Society 1971, 93, 6120-6129.

18. (a) Castro, C.; Karney, W. L.; McShane, C. M.; Pemberton, R. P. [10]Annulene: Bond Shifting and Conformational Mechanisms for Automerization. The Journal of Organic Chemistry 2006, 71, 3001-3006;

(b) Farnell, L.; Kao, J.; Radom, L.; Schaefer, H. F. Structures and stabilities of isomeric [10]annulenes. Journal of the American Chemical Society 1981, 103, 2147-2151; (c) Xie, Y.; Schaefer, H. F.; Liang, G.; Bowen, J. P. [10]Annulene: The Wealth of Energetically Low-Lying Structural Isomers of the Same (CH)10 Connectivity. Journal of the American Chemical Society 1994, 116, 1442-1449; (d) Sulzbach, H. M.; Schaefer, H. F.; Klopper, W.; Lüthi, H. P. Exploring the Boundary between Aromatic and Olefinic Character: Bad News for Second-Order Perturbation Theory and Density Functional Schemes. Journal of the American Chemical Society 1996, 118, 3519-3520; (e) King, R. A.; Crawford, T. D.; Stanton, J. F.; Schaefer, H. F. Conformations of [10]Annulene: More Bad News for Density Functional Theory and Second-Order Perturbation Theory. Journal of the American Chemical Society 1999, 121, 10788-10793; (f) Sulzbach, H. M.; Schleyer, P. v. R.; Jiao, H.; Xie, Y.; Schaefer, H. F. A [10]Annulene Isomer May Be Aromatic, After All! Journal of the American Chemical Society 1995, 117, 1369-1373; (g) Price, D. R.; Stanton, J. F. Computational Study of [10]Annulene NMR Spectra. Organic Letters 2002, 4, 2809-2811.

19. Vogel, E.; Roth, H. D. The Cyclodecapentaene System. Angewandte Chemie International Edition in English 1964, 3, 228-229.

20. (a) Dewey, H. J.; Deger, H.; Froelich, W.; Dick, B.; Klingensmith, K. A.; Hohlneicher, G.; Vogel, E.; Michl, J. Excited states of methano-bridged [10]-, [14]-, and [18]annulenes. Evidence for strong transannular interaction, and relation to homoaromaticity. Journal of the American Chemical Society 1980, 102, 6412-6417; (b) Caramori, G. F.; de Oliveira, K. T.; Galembeck, S. E.; Bultinck, P.; Constantino, M. G. Aromaticity and Homoaromaticity in Methano[10]annulenes. The Journal of Organic Chemistry 2007, 72, 76-85.

21. Evidence for the effect of the sigma framework on these systems can be seen by the colour of 1,6-methano[10]annulene vs 1,5-methano[10]annulene. The former is colourless while the latter is orange (Angew. Chem. Int. Ed., 1981, 20, 1981). The two species also have remarkably different absorption spectra (J. Am. Chem. Soc. 1976, 98, 25, 8277-8279). This is reminiscent of the differences between naphthalene and azulene. This strongly suggests that the sigma framework plays a large role in the electronic structure and must be considered when preparing an ideal [10]annulene.

22. Other cyclodecapentaene structures exist as well (homoazulene: Angew. Chem. Int. Ed., 1981, 20, 1981) and (7b-Methyl-7bH-cyclopent[cd]indene-1,2-dicarboxylic acid:

J. Chem. Soc., Chem. Commun., 1980, 691-692).

23. Billups, W. E.; Blakeney, A. J.; Chow, W. Y. A benzocyclopropene synthesis. Journal of the Chemical Society D: Chemical Communications 1971, 1461-1462.

24. Davalian, D.; Garratt, P. J.; Koller, W.; Mansuri, M. M. Strained aromatic systems. Synthesis of cyclopropabenzocyclobutenes, cyclopropanaphthocylobutenes, and related compounds. The Journal of Organic Chemistry 1980, 45, 4183-4193.

25. It was found that more consistent results could be obtained if the dimesylate was first converted to the diiodide ( $\mathrm{S} 1$, see $\mathrm{SI}$ ) before elimination to the diene (19).

26. The unsymmetric diene was also observed in very small quantities (S2, see $\mathrm{SI}$ ).

27. Moore, W. R.; Ward, H. R. The Formation of Allenes from gem-Dihalocyclopropanes by Reaction with Alkyllithium Reagents1,2. The Journal of Organic Chemistry 1962, 27, 4179-4181.

28. Distillations were attempted using a microscale cold finger. While enrichment of the product was typically observed by $\sim 10 \%$ material loss prohibited further attempts. 
29. The volatility of the product made purification by PTLC especially challenging (see SI).

30. The cyclopropane protons appear as a slightly broadened singlet at $\sim 2.59 \mathrm{ppm}$ (see SI).

31. Castillo, A. M.; Patiny, L.; Wist, J. Fast and accurate algorithm for the simulation of NMR spectra of large spin systems. Journal of Magnetic Resonance 2011, 209, 123-130.

32. Benzene is a highly rigid structure whose $1 \mathrm{H}$-chemical shifts can be replicated through computations without considering rovibrations (see $\mathrm{SI}$ ).

33. (a) Stanger, A. Nucleus-Independent Chemical Shifts (NICS): Distance Dependence and Revised Criteria for Aromaticity and Antiaromaticity. The Journal of Organic Chemistry 2006, 71, 883-893; (b) Schleyer, P. v. R.; Maerker, C.; Dransfeld, A.; Jiao, H.; van Eikema Hommes, N. J. R. Nucleus-Independent Chemical Shifts: A Simple and Efficient Aromaticity Probe. Journal of the American Chemical Society 1996, 118, 6317-6318; (c) Fallah-Bagher-Shaidaei, H.; Wannere, C. S.; Corminboeuf, C.; Puchta, R.; Schleyer, P. v. R. Which NICS Aromaticity Index for Planar $\pi$ Rings Is Best? Organic Letters 2006, 8, 863-866.

34. Geuenich, D.; Hess, K.; Köhler, F.; Herges, R. Anisotropy of the Induced Current Density (ACID), a General Method To Quantify and Visualize Electronic Delocalization. Chemical Reviews 2005, 105, 37583772.

35. Glendening, E. D.; Landis, C. R.; Weinhold, F. Resonance Theory Reboot. Journal of the American Chemical Society 2019, 141, 4156-4166.

36. Natural resonance theory (NRT) computations suggested that the alkynic (44.5\%) and cumulenic (28\%) resonance structures contribute heavily to the wavefunction. Each of the remaining contributions is less than $2.5 \%$. This can be compared to benzene, where the two dominant Kekule resonance structures each contribute $42.5 \%$ to the wavefunction (total: $85 \%$ ). NRT computations were done using NBO6.0 at $\mathrm{M} 062 \mathrm{X} / 6-31+\mathrm{G}(\mathrm{d}, \mathrm{p})$.

37. It is also worth considering the bond lengths of the endo and exo CC bonds in the cyclo propane ring, which are $1.373 \AA$ and $1.374 \AA$ A respectively. Bond angle distortion diagrams also show that fragments corresponding to both resonance structures (divinylcyclopropene and dimethylenecyclopropane) have similar geometric minima, suggesting that bond angles may not favour one resonance structure to a significant degree (see SI).

38. Strain was computed using the StrainViz method (Chem. Sci., 2020,11, 3923-3930, see SI).

39. (a) Siegel, J. S. Mills-Nixon Effect: Wherefore Art Thou? Angewandte Chemie International Edition in English 1994, 33, 1721-1723; (b) Stanger, A. Is the Mills Nixon effect real? Journal of the American Chemical Society 1991, 113, 8277-8280.

40. See SI for mechanism 